\title{
CORRESPONDENCE
}

\section{Neuroendocrine Neoplasms of the Gastrointestinal Tract}

by Prof. Dr. med. Matthias Schott, Prof. Dr. med. Günter Klöppel, PD Dr. med. Andreas Raffel, Prof. Dr. med. Andreas Saleh, Prof. Dr. med. Wolfram Trudo Knoefel und Prof. Dr. med. Werner A. Scherbaum in volume 18/2011

\section{Incomplete Picture}

A review article can be expected to provide a critical appraisal of published scientific evidence, especially if the bibliography is updated to include 2011. This was not done to a sufficient degree in all subchapters on the diagnostic evaluation and treatment of gastroenteropancreatic neuroendocrine tumors (GEP-NET) (1).

The enormous importance of surgical resection undertaken with a curative intent, including surgery to lymph nodes and hepatic metastases in combination with microparticle liver embolization (transcatheter arterial embolization, TAE) and/or radiopeptide therapy in mostly metastasized NET was dealt with to an unsatisfactory extent. Such complex planning is currently the main task of an interdisciplinary NET center and preferably coordinated by an endocrinologist.

Current data on the basis of recent studies do not allow any conclusions about the mean progression time, mean progression-free survival, prolonging the median progression-free interval, and the median time to renewed progression (1). On this background, prolonging the progression-free interval by only six months by means of new molecular therapies (2) is highly unsatisfactory and would presumably hardly be what the authors intended.

The diagnosis of insulinoma in 2011 is based on the biochemical analysis of the unmistakable insulin secretion profile (3). This is recorded for 3-12 hours after glucose loading until neuroglucopenia. Without a secretion profile that is typical for an insulinoma, neuroglucopenia subsequently requires a selective intraarterial calcium injection test (SACI) (4). The 72 hour fasting test or C-peptide suppression test hardly helps to distinguish between patients with insulinoma and patients with pancreatic islet cell hyperplasia.

Pathologists often refer to the latter as nesidioblastosis, whereas clinicians prefer the term non-insulinoma pancreatogenous hypoglycemia syndrome (NIPHS). Measuring proinsulin is vital, in contrast to measuring C-peptides.

Testing for sulfonylureas is uncecessary; it implies that insulinoma patients are dissembling and also means that the indication was not clearly defined. In 30 years' practice, we have never seen any reason to measure sulfonylureas, although we have diagnosed insulinoma in patients with a false-positive glibenclamide test result.

DOI: 10.3238/arztebl.2012.0066a
REFERENCES

1. Schott M, Klöppel G, Raffel A, Saleh A, Knoefel WT, Scherbaum WA: Neuroendocrine neoplasms of the gastrointestinal tract. Dtsch Arztebl Int 2011; 108(18): 305-12.

2. Raymond E, Dahan L, Raoul JL et al.: Sunitinib malate for the treatment of pancreatic neuroendocrine tumors. N Engl J Med 2011; 364: 501-13.

3. Saddig C, Goretzki PE, Starke AAR: Differentiation of insulin secretion patterns in insulinoma. World J Surg 2008; 32: 918-29.

4. Placzkowski KA, Vella A, Thompson GB, Grant CS, Reading CC, Charboneau JW, Andrews JC, Service FJ: Secular trends in the presentation and management of functioning insulinoma at the Mayo Clinic, 1987-2007. J Clin Endocrinol Metab 2009; 94: 1069-73.

Prof. Dr. med. Achim A. R. Starke

Klinik für Endokrinologie und Diabetologie

Universitätsklinikum Düsseldorf

starkea@uni-duesseldorf.de

Prof. Dr. med. Peter E. Goretzki

Klinik für Viszeral- und Endokrine Chirurgie

Lukaskrankenhaus, Neuss

Dr. med. Christiane Saddig

Medizinische Klinik - Hämatologie/Onkologie,

Evangelisches Krankenhaus Düsseldorf

\section{Conflict of interest statement}

Dr Saddig has received expenses from Roche. The other authors declare that no conflict of interest exists.

\section{In Reply:}

In our article we aimed to provide an overview of the numerous and very different gastroenteropancreatic neuroendocrine neoplasms (GEP-NEN). We did not aim to focus exclusively on insulin producing processes and their diagnostic evaluation. However, we will discuss this briefly in what follows.

The European Neuroendocrine Tumor Society (ENETS) guidelines for the diagnostic evaluation of insulinoma stipulate a standardized fasting attempt with preceding glucose loading and recommend measuring insulin and C-peptide. Alternatively to C-peptide, proinsulin may be measured. However, this is not required as an additional test because of the associated costs, which are substantially higher. The calcium stimulation test is done for the purposes of diagnosing the localization, and in patients with unclear hypoglycemia, measuring sulfonylureas is advisable (1).

Sporadic insulinomas have to be differentiated from multifocal insolinomatosis and adult nesidioblastosis, clinically also known as NIPHS (non-insulinoma pancreatic hypoglycemia-syndrome). NIPHS is not, as postulated by Starke et al, the same as pancreatic islet cell hyperplasia, but functional hyperactivity of the beta cells, which can manifest as beta cell hypertrophy. As both conditions have been described in detail only very recently $(2,3)$, clinical guidelines are lacking. 
In our view, the new therapeutics, such as everolimus and sunitinib - for which a prolonged progressionfree interval has been shown, and, in the case of sunitinib, prolonged mean overall survival-represent a clear advance compared with the traditional therapeutic modalities, especially for patients who do not respond to these any more. It goes without saying that these rather new therapeutics will need to be evaluated in further studies, especially in comparison to established therapies.

DOI: 10.3238/arztebl.2012.0066b

\section{REFERENCES}

1. O'Toole D, Grossman A, Gross D et al.: ENETS Consensus Guidelines for the Standards of Care in Neuroendocrine Tumors: biochemical markers. Neuroendocrinology 2009; 90(2): 194-202.
2. Raffel $A$, Krausch MM, Anlauf $M$ et al.: Diffuse nesidioblastosis as a cause of hyperinsulinemic hypoglycemia in adults: a diagnostic and therapeutic challenge. Surgery 2007; 141(2): 179-84.

3. Anlauf M, Bauersfeld J, Raffel A et al.: Insulinomatosis: a multicentric insulinoma disease that frequently causes early recurrent hyperinsulinemic hypoglycemia. Am J Surg Pathol 2009; 33(3): 339-46.

4. Schott M, Klöppel G, Raffel A, Saleh A, Knoefel WT, Scherbaum WA: Neuroendocrine neoplasms of the gastrointestinal tract. Dtsch Arztebl Int 2011; 108(18): 305-12.

\section{On behalf of the authors:}

Prof. Dr. med. Matthias Schott

Universitätsklinik Düsseldorf

matthias.schott@med.uni-duesseldorf.de

\section{Conflict of interest statement}

Professors Schott, Knoefel, and Klöppel have received expenses from Novartis/lbsen. The other authors declare that no conflict of interest exists. 